\title{
Moringa oleifera: un árbol multiusos para las zonas tropicales secas
}

\section{Moringa oleifera: a multipurpose tree for the dry tropics}

\author{
Mark E. Olson ${ }^{1 凶}$ y Jed W. Fahey ${ }^{2}$ \\ 'Departamento de Botánica, Instituto de Biología, Universidad Nacional Autónoma de México. Tercer circuito s/n, Ciudad Universitaria, Apartado \\ postal 70-367, 04510 México, D.F., México. \\ ${ }^{2}$ Johns Hopkins University, School of Medicine, Department of Pharmacology and Molecular Sciences, Lewis B. and Dorothy Cullman Cancer Chemo- \\ protection Center, and Bloomberg School of Public Health, Department of International Health, Center for Human Nutrition. 725 N. Wolfe Street, 406 \\ WBSB Baltimore, Maryland 21205-2185 USA. \\ \molson@ibunam2.ibiologia.unam.mx
}

Resumen. En zonas tropicales secas, el árbol de la moringa (Moringa oleifera Lam.) es objeto de gran atención por parte de los productores, en tanto que crece el número de proveedores que promueven la planta como panacea. Ante esta situación, es necesario separar los usos que están identificados y fundamentados por el conocimiento científico de aquellos que no lo están. Con base en el presente estudio y en la literatura, se presenta un resumen de las bases científicas que sustentan algunos de los beneficios de la moringa, en la medida de lo que hasta hoy se conoce. Los análisis comprueban que la harina de hoja de la moringa se compara favorablemente con la leche en polvo en cuanto a sus componentes de proteína y de calcio y tiene, adicionalmente, un alto contenido de vitamina A. Además de su valor nutritivo, las hojas son ricas en antioxidantes, entre los cuales destacan los isotiocianatos, los cuales parecen presentar propiedades anticancerígenas, hipotensoras, hipoglucemiantes y antibióticas. Las concentraciones de factores antinutritivos en las hojas, tales como inhibidores de proteasas, taninos, saponinas y lectinas, son insignificantes. La mayoría de los estudios sobre las cualidades benéficas de la moringa se han llevado a cabo in vitro o en animales; por lo tanto, se desconocen las dosis necesarias para producir algún efecto benéfico en humanos. Sin embargo, dado que los beneficios potenciales son muchos y los niveles de sustancias antinutricionales bajísimos, no encontramos argumentos en contra del consumo de la planta. En resumen, Moringa oleifera es un alimento nutritivo y benéfico que ofrece características muy atractivas para establecer su cultivo en comunidades sostenibles en el trópico seco de México y otros países de Latinoamérica.

Palabras clave: factores antinutricionales, antioxidantes, desarrollo comunitario, germoplasma, glucosinolatos/ isotiocianatos, nutrición, proteínas, agricultura tropical.

\begin{abstract}
Farmers in the dry tropics are increasingly turning their attention to the moringa tree (Moringa oleifera Lam.), while the plant is increasingly touted as a cure-all for ailments from cancer to hysteria. Given the interest in the plant, it is crucial to separate unsubstantiated uses from scientific understanding. Drawing on our own research and on the literature at large, we summarize the scientific basis for several of moringa's purported benefits while emphasizing the limits to our knowledge. Nutritional analyses show that dried moring leaf powder compares favorably with powdered milk in its protein and calcium content, in addition to having a high content of Vitamin A. In addition to their nutrients, moringa leaves contain powerful antioxidants, of which the isothiocyanates are remarkable for their anticancer, hypotensive, hypoglycemic, and antibiotic properties. At the same time, antinutritional factors, such as protease inhibitors, tannins, saponins, and lectins are found in very low amounts. The great majority of studies on moringa properties have been carried out in vitro or in animal systems. Without clinical studies in humans, it is impossible to specify the dose necessary to produce a specific beneficial effect in humans. However, the levels of antinutritional factors are sufficiently low and the potential benefial effects are sufficient that we find no arguments against consumption of the plant. In summary, as a nutritious and beneficial food, Moringa oleifera appears to offer an attractive component for building sustainable communities in the in dry tropics of Mexico and other parts of Latin America.
\end{abstract}

Key words: antinutritional factors, antioxidants, community development, germplasm, glucosinolates/isothiocyanates, nutrition, protein, tropical agriculture. 


\section{Introducción}

La importancia del trópico seco para la humanidad, junto con la urgente necesidad de su conservación, hace esencial el estudio, la ampliación y optimización de la gama de especies vegetales disponibles para el aprovechamiento de estas zonas (Janzen, 1988; Miles et al., 2006; Stephenson and Fahey, 2004; Piperno et al., 2009). En este contexto, un árbol que ha recibido mucha atención en los últimos años (Fuglie, 2001; Fahey, 2005; Ferreira et al., 2008) es Moringa oleifera Lam., conocido comúnmente como moringa. Este árbol tiene un gran potencial para su cultivo en México así como en muchas partes de América tropical por su combinación singular de propiedades. Las hojas son comestibles y ricas en proteínas, con un perfil de aminoácidos esenciales muy balanceado. Al mismo tiempo, contiene vitaminas, principalmente $\mathrm{A} y$ $\mathrm{C}$, en altas cantidades, así como antioxidantes potentes. Los frutos jóvenes son comestibles y las semillas producen un aceite comestible y lubricante de altísima calidad. Los desechos del prensado de las semillas para obtener el aceite contienen uno de los floculantes o aglutinantes vegetales más potentes que se conocen y pueden eliminar la turbidez del agua. Sus hojas ofrecen un forraje nutritivo para los animales, así como también los residuos de las semillas después de la extracción de aceite y aun las ramas molidas (Martínez, 1959; Reyes et al., 2006). Estos son sólo algunos de los usos de este árbol, que además crece con suma rapidez, tolera el calor y es resistente a las sequías.

A pesar de su auge notable, existen muchas dudas acerca de la moringa, tanto entre los productores potenciales como en los consumidores. Muchas de estas dudas surgen del escepticismo que inevitablemente generan las campañas publicitarias que bombardean al público con una panacea tras otra en el intento de generar ganancias. El aumento en la oferta de productos derivados de la moringa ejemplifica este fenómeno, y en su publicidad podemos encontrar afirmaciones de que no sólo es nutritiva, sino que curará desde la epilepsia hasta la histeria. Asimismo, muchas personas se preguntan si el consumo de la moringa podría tener algún efecto colateral. El propósito de este artículo es ofrecer una introducción breve a este árbol tan singular. Posteriormente, se realiza una breve revisión de los beneficios que se le atribuyen a la moringa y se intenta identificar hasta qué punto estas aplicaciones están sustentadas por trabajo científico. Al mismo tiempo, se precisan algunos de los aspectos que requieren mayor investigación. Se muestra por qué la moringa es un árbol útil cuyo consumo ofrece beneficios importantes, pero lejos de presentarlo como una panacea, se sugiere que la comercialización de sus productos tiene que ser clara respecto a lo que genuinamente ofrece la planta, y conforme al nivel de conocimiento que de ella se tiene.

Introducción a las moringáceas. La moringa pertenece a la familia Moringaceae, un grupo pequeño de plantas dentro del inmenso orden Brassicales que incluye la familia de la col y del rábano, junto con la familia del mastuerzo y de las alcaparras (APG, 2009). La familia más cercanamente emparentada con Moringaceae es Caricaceae, la de la papaya, con la cual comparte la característica de presentar glándulas en el ápice del peciolo (Fig. 1; Olson, 2002b). Moringaceae comprende únicamente un género, Moringa. Dentro de Moringa hay 13 especies (Verdcourt, 1985; Olson, 2002a), las cuales abarcan una gama muy diversa de hábitos o formas de crecimiento, desde hierbitas y arbustos hasta árboles grandes (Olson y Razafimandimbison, 2000; Olson, 2001a y 2001b ). Si bien varían mucho en su forma, es muy fácil distinguir un miembro de Moringa de cualquier otra planta.

Moringaceae se distingue de las otras familias por una combinación única de rasgos (Olson, 2010). Sus especies se caracterizan por tener hojas pinnadas grandes, en donde cada hoja está dividida en muchos folíolos dispuestos sobre un armazón llamado raquis (Fig. 1A). Los frutos forman una cápsula larga y leñosa que cuando alcanza la madurez se abre lentamente en 3 valvas que se separan la una de la otra por su longitud, quedando pegadas sólo en la base del fruto (Figs. 1B, C). En la mayoría de las especies, las semillas presentan 3 alas longitudinales. La combinación de hojas pinnadas, frutos trivalvados y semillas con 3 alas hace que sea muy fácil reconocer una Moringa. Para asegurar la identificación, se pueden buscar las glándulas foliares características de esta familia, las cuales se encuentran en ambos lados flanqueando la base o en el ápice del pecíolo y en la mayoría de las articulaciones del raquis (Fig. 1). Otras características únicas de la familia, pero menos fáciles de observar, incluyen el estilo hueco y las anteras con 2 esporangios o cámaras para el polen en vez de los 4 que suelen presentar las plantas con flor (Olson, 2003). Características aún menos aparentes incluyen los ductos de goma en la médula de los tallos y elementos de vaso con placas de perforación sin bordes (Olson y Carlquist, 2001; Olson, 2002b).

Moringa oleifera: nombres y distribución. Si bien la moringa es fácil de reconocer, existe confusión acerca de cuál es su nombre científico correcto (Keraudren y Gillett, 1963). A la planta que conocemos como Moringa oleifera se le han aplicado nombres como Guilandina moringa, que se remonta a Linneo en el año 1753, y también Hyperanthera moringa (L.) Vahl. Todavía es común que algunos autores empleen el nombre Moringa pterygosperma Gaertn. (p.ej. Morton, 1991), que es un nombre ilegítimo de acuerdo con las reglas de nomenclatura botánica. Estas reglas también 

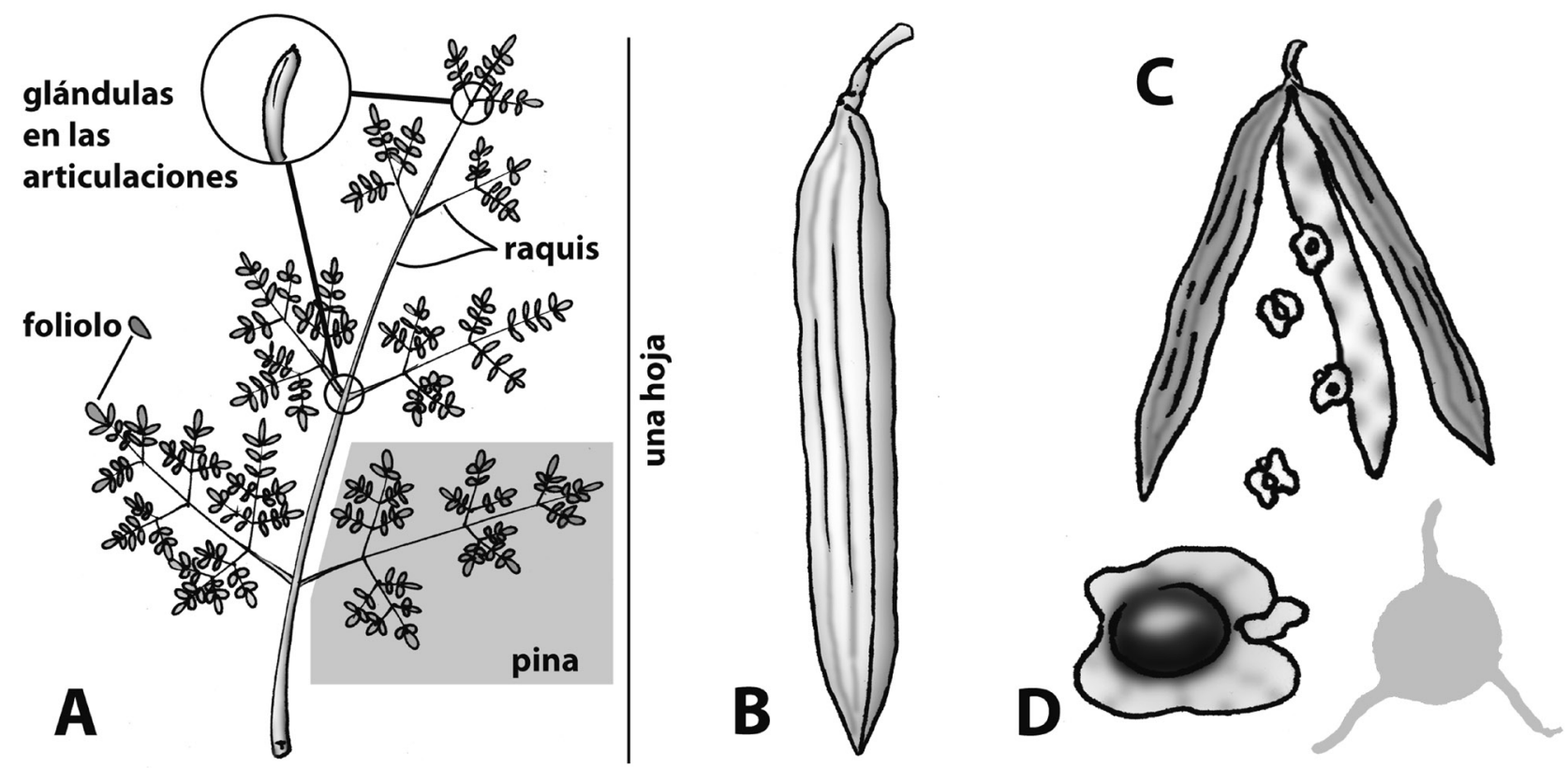

Figura 1. Identificación. La moringa (Moringa oleifera) es fácil de identificar por su combinación inconfundible de caracteres. A, hojas grandes, pinnadas, que pueden alcanzar unos $60 \mathrm{~cm}$ de longitud; están divididas en folíolos dispuestos sobre un raquis. En la articulación de cada raquis se encuentran pequeñas glándulas de $1 \mathrm{~mm}$ de longitud. B-D. Frutos y semillas. B, fruto, una cápsula ligera, leñosa y seca, que en la madurez mide de 10 a 30 o hasta $50 \mathrm{~cm}$; C, el fruto se abre en 3 partes o valvas; D, semillas de 1.5-3 cm de diámetro con un centro de color café oscuro y 3 alas de color beige; la silueta muestra la configuración de las 3 alas. La moringa es la única planta en México con hojas pinnadas con glándulas en las articulaciones, frutos con 3 valvas y semillas con 3 alas.

indican que G. moringa y H. moringa carecen de validez, mientras $M$. oleifera tiene prioridad y constituye el nombre válido. Para fines de la presente discusión emplearemos "moringa" y "Moringa oleifera" como términos equivalentes mientras "Moringa" se referirá al género.

Otros de los problemas que enfrenta esta especie es el conocimiento de su historia natural, ya que, como muchas plantas de importancia económica, se sabe muy poco de Moringa oleifera en el estado silvestre. Se ha registrado en los bosques tropicales caducifolios del noroeste de la India y en el este de Pakistán (p.ej. Haines, 1922), en la zona entre Simla en la India y Faisalabad en Pakistán (Verdcourt, 1985). Sin embargo, existen pocos registros publicados acerca de la distribución natural de la planta, por lo cual un estudio detallado de los bosques remanentes de esa zona revelaría mucho acerca de la distribución natural de este recurso importante, así como del germoplasma existente (Olson, 2001b). Pandey et al. (2010) presentan un mapa de distribución que muestra una franja amplia del norte de la India donde la planta crece silvestre pero, lamentablemente, no ofrecen una discusión sobre cómo se distinguen los árboles silvestres de los cultivados, una consideración esencial para poder dilucidar la distribución natural de una planta cultivada. Por lo tanto, seguimos sin entender bien la distribución silvestre de esta planta. En contraste con lo poco que se sabe acerca de su distribución natural, queda ampliamente comprobado por registros de herbario que $M$. oleifera se cultiva en todos los países tropicales del mundo (Verdcourt, 1985).

Cuando se habla de la distribución de la moringa es esencial hacer la distinción entre términos como "nativo", "silvestre" y "naturalizado". Varios autores que escriben sobre los usos de M. oleifera se refieren a la planta como "naturalizada" o hasta "silvestre" cuando se observa en países fuera de su lugar de origen. Esta terminología es incorrecta, pues el proceso de naturalización biológica implica que un organismo se establezca en una región en donde no es nativo y logre sobrevivir y reproducirse por muchas generaciones sin asistencia humana. Estas plantas se llaman malezas y pueden llegar a ser un problema sumamente serio para la agricultura y para el manejo de recursos naturales silvestres (Villaseñor y Espinoza, 1998). Si una planta llega a encontrarse en una lista de malezas conocidas, sus posibilidades de transporte entre países y su utilización podrían verse severamente restringidos (véase la reglamentación australiana, que prohibe la importación de M. stenopetala a ese país). En el caso de M. oleifera, no existen registros de su naturalización en ningún hábitat natural (Olson, 2010). A veces se pueden encontrar plántulas estableciéndose debajo de sus progenitores en zonas 
perturbadas, tales como lotes baldíos, camellones y bordes de caminos, pero esta es una situación muy distinta a, por ejemplo, la invasión en los desiertos de México por el pasto Cenchrus ciliaris, que sí está naturalizado y que tiene un impacto sumamente nocivo en estas comunidades (de la Barrera, 2008). Para evitar restricciones innecesarias en cuanto al transporte y la utilización de la moringa, es importante evitar términos como "naturalización" o "silvestre" cuando se refiere a la práctica de cultivar la planta. Esto no implica que no sea necesario proceder con cautela con cualquier planta no nativa, pero en el caso de M. oleifera, no se cuenta con ningún registro de la planta como maleza en ninguna parte del mundo, únicamente registros de cultivo muy extensos.

Muchos productores agrícolas en México se preguntan si la planta se puede cultivar en este país. La respuesta es que ya es parte de la horticultura tradicional desde hace mucho tiempo, principalmente con fines ornamentales: la encontramos abundantemente en los pueblos de toda la costa del Pacífico, desde el sur de Sonora hasta Chiapas, incluyendo el sur de la península de Baja California (al sur de La Paz y de Todos Santos). Los ejemplares de moringa son especialmente abundantes y frondosos en las llanuras calientes del sur del istmo de Tehuantepec. La planta también se cultiva en los poblados de las depresiones tropicales secas del país, como la del Balsas y la depresión central de Chiapas. La planta se encuentra en los pueblos de la zona del Infiernillo y en las cercanías de Apatzingán, Mezcala, Iguala y Tequesquitengo. Como se puede apreciar gracias a su distribución cultivada, la moringa es una planta de zonas cálidas que nunca sufren heladas. En general, prospera mejor por debajo de los $500 \mathrm{~m}$ snm y crece muy poco cuando se cultiva a altitudes mayores a 1500 metros.

Es probable que la planta haya llegado a territorio mexicano por primera vez gracias a marineros filipinos durante los viajes de la Nao de China, que cubría la ruta entre Manila y Acapulco. Si llegó de esta manera, seguramente era utilizada como alimento por los miembros de la tripulación. Este hábito de comer la planta se ha perdido a lo largo de los siglos, pues como se mencionó anteriormente, las plantas en cultivo informal en México tienen casi exclusivamente fines ornamentales (observación de los autores). Sin embargo, en 1959, Martínez anotó que "las vainas tiernas son comestibles y se usan en sopa o se preparan a manera de espárragos; las raíces tienen sabor picante como el rábano rústico y se usan como condimento en lugar de éste, las semillas maduras se tuestan y consumen como nueces, siendo su sabor dulce, ligeramente amargo y agradable; las almendras son oleaginosas; las hojas se comen como verdura y también pueden servir de forraje" (p. 415). Como evidencia del interés en la producción de esta planta desde el siglo pasado, este mismo autor comenta que "El Ferrocarril del Sud Pacífico, por conducto de su Departamento Industrial y Agrícola hizo algunos trabajos de introducción y aclimatación en Escuinapa, Rosario y Culiacán, Sin. y en Empalme, Son.” (p. 416).

Con el reciente auge mundial del cultivo de la moringa (Fuglie, 2001), el árbol ha llegado a México en forma de semillas desde África y la India, generalmente para su cultivo en campos especializados, con la finalidad de cosechar hojas. Si bien actualmente hay en muchos países un gran interés en el aprovechamiento del árbol y la planta ha existido en México quizás por siglos, las personas que practican el cultivo popular de $M$. oleifera usualmente desconocen el interés por el árbol, mientras los agricultores interesados en cultivar la planta a escalas grandes ignoran la presencia de la moringa en la horticultura tradicional mexicana (véase también Thurber y Fahey, 2009).

Propiedades de Moringa oleifera y su sustento cientifico. Proteínas. Una de las características más atractivas de la moringa es el alto contenido de proteína en sus hojas. Los testimonios de Fuglie (2001) sobre un sinfín de casos en África occidental donde la adición de moringa a la dieta rescató a personas en desnutrición extrema se han tomado como evidencia del extraordinario valor del contenido proteínico de la planta. En este sentido, sus beneficios nutricionales son tan ampliamente reconocidos que hay poco lugar para dudar del impacto positivo del consumo de harina de hoja de moringa en situaciones de inanición inminente. Sin embargo, el desarrollo de un mayor número de pruebas clínicas bien controladas y documentadas con claridad sería de inmenso valor. Los análisis del contenido proteínico de las hojas secas muestran que hasta el $30 \%$ de su peso está formado por proteína (la leche en polvo contiene $35 \%$ ) y que la mayor parte de ésta parece ser directamente asimilable. Además, las hojas contienen todos los aminoácidos esenciales (las unidades de las proteínas que el cuerpo no puede sintetizar) en un perfil alto y bien balanceado (Freiberger et al., 1998). Por todo esto, es claro que la moringa es un alimento importante, un hallazgo que ha sido comprobado de manera repetida (p.ej. Richter et al., 2003). Muchas plantas muestran estructuras ricas en proteínas, por ejemplo los frijoles. Sin embargo, mientras la mayoría de ellas producen estas proteínas en sus frutos, la moringa se destaca por contener las proteínas en sus hojas, las cuales están presentes en el árbol prácticamente todo el año.

Calcio y vitamina A. Varios estudios han indicado que la moringa es una fuente valiosa de vitamina $\mathrm{A}$, pero ha permanecido la duda de si el contenido de esta vitamina se conserva aún después del secado y molido de la hoja. Para examinar esta interrogante, Nambiar y Seshadri (2001) alimentaron ratas con una dieta sin vitamina A por 4 semanas, un tratamiento tan extremo que 4 de las 40 ratas murieron. 
Al término de las 4 semanas, los autores dividieron las ratas en 4 grupos. Un grupo recibió acetato de vitamina A, el segundo grupo hoja fresca de moringa, el tercer grupo hoja deshidratada y el cuarto grupo sirvió como comparación y siguió con la dieta carente de vitamina A. Después de 4 semanas, encontraron que, si bien los niveles sanguíneos de vitamina A fueron un poco más bajos en las ratas suplementadas con moringa en comparación con aquellas que recibieron acetato de vitamina A (25.8-28.2 $\mu \mathrm{g} / \mathrm{dL}$ vs. $34.7 \mu \mathrm{g} / \mathrm{dL}$ ), la administración de moringa parece ser más que suficiente para contrarrestar los efectos de la falta de vitamina A y a una fracción del costo del acetato de vitamina A. Además, el grupo que adquirió más peso fue aquel que consumió moringa, posiblemente por el contenido de proteína en las hojas. En el estudio de Nambiar y Seshadri (2001) quedó claro que tanto las hojas frescas como las secas sirven como suplemento de vitamina A. Más notable aún es el hallazgo de Seshadri et al. (1997), quienes mostraron que las hojas de moringa deshidratadas conservaron su contenido de vitamina A después de 90 días de almacenamiento. Cabe mencionar que es probable que no cualquier proceso de secado conserve las propiedades de las hojas. Es casi seguramente esencial secar las hojas en la sombra y sin exposición a la luz ultravioleta artificial o del sol, pues esta luz fragmenta las largas cadenas moleculares de la vitamina (Allwood y Plane, 1984).

Si bien estudios como el de Nambiar y Seshadri (2001) apuntan fuertemente a la utilidad de la moringa como fuente de vitamina A, escasean los estudios en seres humanos. Un dato notable en esta dirección es el trabajo de Fernández (2010), en donde se suplementó la dieta de 31 niños preescolares de 3 y 5 años de edad con 1.17 gramos de harina de hoja de Moringa al día durante 5 meses. Los resultados se compararon contra un grupo control de 25 niños que no recibieron el suplemento alimenticio. $\mathrm{Si}$ bien al inicio del estudio no se encontraron diferencias en cuanto al nivel de nutrición de los niños, la autora encontró que la prevalencia de deficiencia de vitamina A disminuyó significativamente de 40 a $14.3 \%$ en los niños que recibieron la hoja de Moringa, mientras que el grupo de los niños que no recibieron suplemento permanecieron estadísticamente con la misma prevalencia de deficiencia. Asimismo, se observó un aumento notable en la concentración de hemoglobina (la molécula de la sangre que porta el oxígeno) en los niños que recibieron Moringa. Estos resultados brindan apoyo a la eficacia de la moringa para combatir la desnutrición humana.

Además de la vitamina A, se suele decir que las hojas de la moringa contienen altos niveles de calcio. En la literatura popular, y especialmente en la publicidad, se leen una y otra vez afirmaciones sobre el alto contenido de calcio de la moringa, tales como "la moringa contiene más calcio por gramo que el yogurt." Si bien es cierto que existe un alto contenido de calcio, una parte importante de este calcio está como cristales de oxalato de calcio en las células de la planta (Olson y Carlquist, 2001; Olson, 2001a), una forma que el cuerpo no puede asimilar y que es excretada directamente. Radek y Savage (2008) cuantificaron que el porcentaje de calcio en la moringa en forma de oxalato, y que no puede ser absorbido, es de casi el 38\%. Esta cantidad podría parecer muy alta, pero también mostraron que la moringa tiene niveles sumamente altos de calcio $(>20 \mathrm{mg} / \mathrm{g}$ de hoja seca), por lo que aún con la tercera parte en una forma no asimilable, la moringa ofrece cantidades notables de calcio a la dieta. La leche en polvo contiene alrededor de $13 \mathrm{mg} / \mathrm{g}$ de calcio (USAID, 2006). Por lo tanto, el polvo de moringa parece compararse favorablemente no sólo en cuanto a su contenido de proteína con la leche en polvo, sino que también en cuanto a su contenido de calcio, a un costo de producción notablemente más bajo que la leche y con un impacto ambiental mucho menor.

Prevención y tratamiento de enfermedades. Los beneficios que se pueden percibir en cuanto al tratamiento o la prevención de enfermedades, y en cuanto al tratamiento de infecciones a través de la aplicación de preparados de moringa, no están tan bien entendidos como sus beneficios nutritivos (Palada, 1996). Si bien existe una tradición extensa, y los testimonios sobre sus beneficios médicos son voluminosos, estos beneficios han recibido relativamente poca investigación científica. Por lo tanto, aquí se intenta revisar algunos de los principales beneficios que se le han atribuido a la planta y la calidad y naturaleza de la evidencia disponible. Es muy recomendable la lectura de 2 artículos recientes que equilibran la evidencia derivada de la medicina no convencional, tales como la medicina tradicional, el conocimiento tribal y testimonios personales, con las pruebas científicas, las cuales son necesarias para tomar decisiones sobre la eficacia de estas prácticas (Sampson, 2005; Talalay y Talalay, 2001).

Desafortunadamente, mucha de la información sobre el efecto de las propiedades de la moringa en seres humanos no cuenta con evidencia apoyada en pruebas clínicas aleatorizadas y controladas con placebo, y tampoco se ha publicado en revistas científicas de alta circulación. Por ejemplo, Shaw y Jana (1982) parecen establecer la moringa como una cura poderosa contra infecciones urinarias; sin embargo, este estudio no incluyó en su diseño algún tipo de comparación o grupo control. Los estudios que carecen de comprobación son rechazados por la medicina occidental, por lo que las propiedades medicinales o nutricionales de la moringa no serán aceptadas por los médicos occidentales hasta que se lleven a cabo estudios de este tipo.

En muchos casos, los estudios se han basado en investigaciones in vitro (en células en cultivo en el laboratorio) o 
bien han empleado animales como objeto de estudio. Estos estudios aportan explicaciones mecanicistas para algunos de los supuestos beneficios que han surgido de la medicina tradicional (Luqman et al., 2009). Por ejemplo, muchos de estos estudios han comprobado que el tratamiento con la moringa o sus extractos eleva los niveles de una variedad de biomarcadores, antioxidantes y enzimas de destoxificación (Fahey et al., 2004; Faizi et al., 1994; Kumar y Pari, 2003; Rao et al., 1999). Aquí trataremos brevemente el efecto antibiótico y de prevención de cáncer como 2 ejemplos de investigación sobre la moringa en donde la evidencia parece ser especialmente robusta.

Actividad antibiótica. En apoyo de esta propiedad de la moringa, la evidencia tanto científica como tradicional es abrumadora. A pesar de que la evidencia científica ha estado disponible por más de 50 años, la mayoría de los médicos occidentales la desconocen. A finales de la década de 1940 e inicios de la de 1950, un equipo de científicos en la India identificaron una sustancia que llamaron pterigospermina (Das et al., 1954, Anderson et al., 1986), un compuesto que se disociaba fácilmente en 2 moléculas de isotiocianato bencílico (Kurup y Narasimha Rao, 1952, 1954a, b y c; Das et al., 1957a, 1957b; véase también Fahey et al., 2001; Bennett et al., 2003; Amaglo et al., 2010). Ya en ese entonces se intuía que el isotiocianato bencílico tenía un efecto antimicrobiano. Este grupo de científicos no sólo identificó la pterigospermina sino que a la mitad de la década de 1950 llevó a cabo caracterizaciones detalladas y elegantes de la modalidad específica de su actividad antimicrobiana. Investigaciones posteriores también mostraron que la pterigospermina y extractos de la moringa presentaron actividad antibiótica en contra de una variedad de microbios. Sin embargo, el aislamiento y la caracterización de la estructura de estas moléculas resulta un reto técnico, pues pueden ser fácilmente dañadas durante la manipulación. Un ejemplo de estas dificultades podría ser precisamente el caso de la pterigospermina, pues la identificación original de esta sustancia ha sido cuestionada, atribuyéndose a un componente del aislamiento o errores en la caracterización estructural (Eilert et al., 1981). Esto ilustra el hecho de que queda mucho todavía por aprender acerca de este árbol singular. En 1964, Bennie Badget, estudiante del famoso químico Martin Ettlinger, publicó en su tesis doctoral los análisis elegantes y cuidadosos con los que identificó una serie de compuestos derivados del isotiocianato bencílico. La identidad de estos compuestos, sin embargo, no estuvo disponible en la literatura científica general hasta su "redescubrimiento", 15 años después, por parte de Kjaer y colaboradores (1979). Posteriormente, se llevaron a cabo estudios fundamentales sobre la actividad antibiótica del 4-( $\alpha$-L-ramnopiranosiloxi) bencil glucosinolato (Fig. 2F) y su isotiocianato correspondiente (Fig. 2B). Dichas prue- bas se destacan por haber demostrado la actividad de esta sustancia contra una gama muy amplia de bacterias y hasta de hongos.

Trabajos recientes de miembros del equipo de los autores se han enfocado en determinar la actividad del 4-(-L-ramnopiranosiloxi) bencil isotiocianato, del bencil isotiocianato y otros isotiocianatos contra la bacteria Helicobacter pylori. A pesar de que esta bacteria se descubrió tan sólo en la década de 1980, H. pylori es un patógeno humano omnipresente en zonas pobres del mundo y otras áreas con cuidado médico insuficiente. Es una de las causas principales de la gastritis y de úlceras gástricas y duodenales y además representa un factor de riesgo muy fuerte para cáncer de estómago, siendo clasificado como un factor cancerígeno por parte de la Organización Mundial para la Salud desde 1993. En cuanto a la eficacia de la moringa, cultivos en el laboratorio de H. pylori resultaron ser extraordinariamente susceptibles al 4-(-L-ramnopiranosiloxi) bencil isotiocianato y a una variedad de otros isiotiocianatos (Fahey et al., 2002; Haristoy et al., 2005). Estos compuestos presentaron actividad contra $H$. pylori en concentraciones hasta 1000 veces más bajas que aquellas que se emplearon en estudios previos. La actividad de la moringa en contra de H. pylori se está estudiando actualmente en pruebas clínicas y el isotiocianato está mostrando actividad apreciable en estudios pilotos (Galan et al., 2004; Yanaka et al., 2005).

Prevención del cáncer. Existe la práctica en la medicina tradicional de emplear la moringa para tratar tumores (Hartwell, 1967-1971). Por lo tanto, miembros del equipo que llevó a cabo este estudio, investigaron la actividad del 4-(4'- $O$-acetil- $\alpha$-L-ramnopiranosiloxi) bencil isotiocianato y 4-(-L-ramnopiranosiloxi) bencil isotiocianato contra el cáncer. En un estudio de células cultivadas en el laboratorio, se demostró que el 4-(4'-O-acetil- $\alpha$-Lramnopiranosiloxi)bencil isotiocianato y el compuesto relacionado niacimicina inhiben fuertemente la inducción de los antígenos que se asocian con ciertos tipos de cáncer (véase también Guevara, et al., 1999; Murakami et al., 1998). Asimismo, la niacimicina también inhibió la producción de tumores en ratones que recibieron dosis de sustancias cancerígenas, mientras el grupo control fue afectado por numerosos tumores (Murakami et al., 1998). En otro estudio en ratones, Bharali et al. (2003) examinaron la prevención de tumores de la piel tras el consumo de extractos de los frutos de la moringa. Este estudio incluyó controles tanto negativos como positivos y mostró una reducción dramática en papilomas de la piel en el grupo que consumió la moringa.

Las prácticas médicas tradicionales han ofrecido indicios desde hace mucho tiempo de que plantas como la moringa podrían desempeñar un papel clave tanto en la pre- 

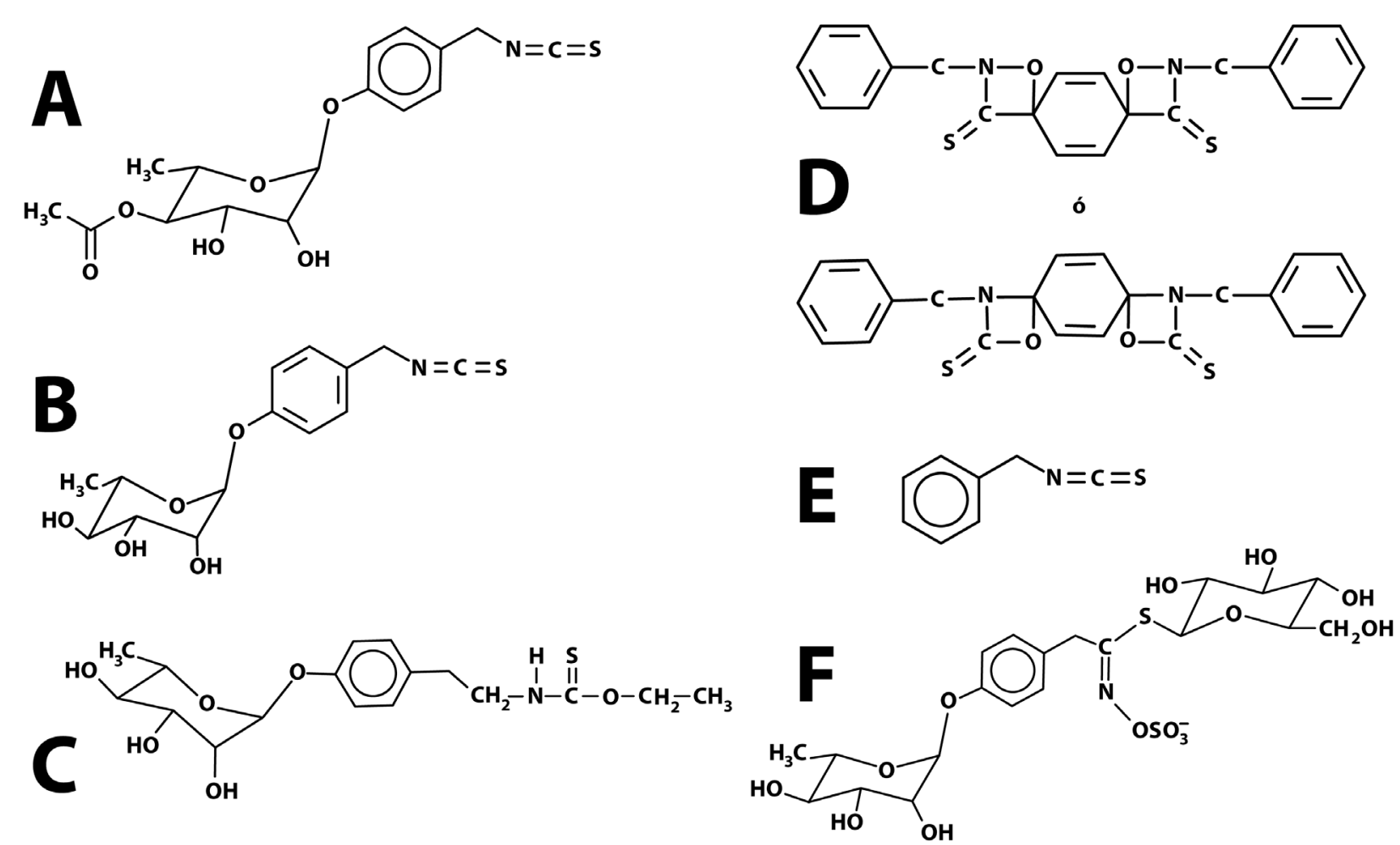

Figura 2. Estructuras fitoquímicas importantes en las especies de Moringa. A, 4-(4'-O-acetil- $\alpha$-L-ramnopiranosiloxi) bencil isotiocianato; B, 4-(-L-ramnopiranosiloxi) bencil isotiocianato; C, niacimicina (niazimicin en inglés); D, pterigospermina; la identidad de la pterigospermina se ha puesto en duda en estudios que sugieren que podría representar un artefacto del proceso de aislamiento o bien un error de la determinación estructural; E, bencil isotiocianato; F, 4-( $\alpha$-L-ramnopiranosiloxi) bencil glucosinolato.

vención del cáncer como en terapias para su tratamiento. A su vez, investigaciones recientes han aislado compuestos bioactivos de extractos de moringa. Desafortunadamente, hasta la fecha, las pruebas que exige la medicina moderna no se han realizado. Esto se debe en gran medida a que no se ha demostrado el efecto preventivo contra el cáncer ni la presencia de biomarcadores indicadores de un estado protegido en el ser humano. ¿Significa esto que la moringa no es un quimioprotector? No. Es perfectamente posible que sí lo sea, pero se requiere de estudios más rigurosos para alcanzar la plena aceptación de la moringa como una herramienta para la prevención del cáncer.

Colesterol y glucosa. Algunos usos tradicionales sugieren que la moringa podría ser útil tanto para bajar los niveles de colesterol como los de la glucosa. Estudios recientes en animales parecen sustentar estos usos populares. Por ejemplo, Mehta et al. (2003) alimentaron conejos por 4 meses de una dieta alta en colesterol, provocando altos niveles en la sangre de los animales. Al cabo de 4 meses, incluyeron una dosis de $200 \mathrm{mg}$ de frutos inmaduros (cocidos, secados y molidos) por kilo de peso de cada conejo. Este tratamiento resultó en una reducción del colesterol sanguíneo en comparación con los animales que no recibieron frutos de moringa, así como una reducción en lípidos tota- les y triglicéridos. Al mismo tiempo, los conejos con altos niveles de colesterol total sufrieron un cambio reduciendo sus lipoproteínas de baja densidad ("colesterol malo") e incrementando las lipoproteínas de alta densidad ("colesterol bueno").

La regulación de los niveles de glucosa (Kar et al., 2003) en México, como en otras partes del mundo, es de gran interés por la incidencia alta de diabetes. Por ejemplo, Ndong et al. (2007) llevaron a cabo un estudio que incluyó tanto ratones normales como ratones Goto-Kakizaki, que presentan diabetes tipo II sin presentar obesidad. Administraron a los ratones glucosa, con y sin harina de hoja de moringa y encontraron que los niveles de glucosa en los ratones que habían comido moringa no fueron tan altos como los de los ratones que no la comieron. Los autores sugirieron que la actividad regulatoria de glucosa de la moringa podría darse como resultado de su alto contenido de sustancias polifenólicas como la quercetina y el kempferolo. Existe evidencia, entonces, que respalda a la moringa como una alternativa de bajo costo para la regulación de los niveles de glucosa en la sangre.

Antioxidantes en alimentos El proceso de la oxidación no sólo causa la corrosión del hierro sino que procesos parecidos también afectan los alimentos. Los agentes de 
conservación se agregan a éstos precisamente para que las grasas poliinsaturadas, como son los aceites vegetales, oxiden (arrancien) más lentamente. Estudios recientes han explorado extractos de la moringa como una alternativa natural a los conservadores artificiales. Anwar et al. (2007) prepararon varios extractos de las hojas de moringa, agregaron los extractos a aceite de girasol y lo almacenaron por 2 meses. Pasado este tiempo, compararon los niveles de peróxidos, dienos y trienos conjugados y los valores de p-anisidina, todos cambios moleculares producidos al arranciarse los aceites. Increíblemente, en los aceites sin el extracto de moringa estos indicadores estaban presentes al doble en comparación con los aceites con moringa. De la misma forma, Reddy et al. (2003) observaron que una cantidad pequeña de hoja molida de moringa retrasó significativamente el proceso de arranciamiento en galletas. Otros estudios comprobaron el efecto antioxidante de las hojas deshidratadas no molidas (Siddhuraju y Becker, 2003) y que el poder antioxidante de las hojas es mayor que el de los frutos verdes o las semillas (Singh et al., 2009).

A partir de estudios como estos, queda claro que $M$. oleifera ofrece antioxidantes eficaces que podrían brindar agentes conservadores naturales alternativos a los conservadores tradicionales como el BHA y BHT. Sin embargo, es posible que no todos los individuos de moringa tengan la misma eficacia. Por ejemplo, Siddhuraju y Becker (2003) mostraron que la actividad antioxidante de distintas cepas de $M$. oleifera cultivadas en la India, en Níger y en Nicaragua difirió en las cantidades relativas de las diferentes sustancias antioxidantes y en su actividad antioxidante en general (véase también Doerr et al., 2009). Por lo tanto, queda claro que es necesario contar con la mayor cantidad posible de cepas de $M$. oleifera y un mayor conocimiento de la variación en los parámetros de interés entre ellas.

¿Tiene efectos colaterales la moringa? Sustancias antinutricionales Muchas personas preguntan cuáles podrían ser los peligros asociados al consumo de la moringa, por lo que en esta sección se abordan algunas cualidades "antinutritivas". Muchas plantas contienen una buena cantidad de proteína o vitaminas, pero las sustancias antinutritivas que también pueden llegar a tener vuelven su consumo desagradable o hasta peligroso. Por ejemplo, el mesquite contiene cantidades importantes de ciertas proteínas, pero también inhibidores de tripsina (Ortega-Nieblas et al., 1996). La tripsina es un tipo de proteasa, es decir, una enzima que digiere proteínas. Los inhibidores de las proteasas interfieren con la digestión y su presencia en las plantas podría servir para ahuyentar a los herbívoros en el ambiente natural de la planta. Otras sustancias antinutritivas incluyen los taninos, las sustancias amargas que tiñen la corteza de los árboles de color café. Los taninos son compuestos que se unen fuertemente a las proteínas, convirtiéndolas en no digeribles. Por su parte, las saponinas, sustancias de comportamiento "jabonoso" que también son amargas y a veces tóxicas, son glucósidos que disminuyen la tensión superficial en soluciones acuosas, produciendo una espuma estable y también son capaces de hemólisis (desintegración de los glóbulos rojos). Finalmente, las lectinas son glicoproteínas que se unen a los mucopolisácaridos de la pared intestinal y se pueden considerar como auténticas enterotoxinas. La moringa se destaca porque, a pesar de su alto contenido de proteínas y vitaminas, contiene muy bajos niveles de sustancias antinutritivas. Makkar y Becker (1996) mostraron que las hojas de moringa contenían cantidades despreciables de taninos; asimismo, sus análisis no arrojaron indicios ni de lectinas ni de inhibidores de tripsinas. Encontraron saponinas, pero en cantidades bajas, más o menos equivalentes a los niveles registrados en los frijoles de soya, es decir, en niveles inocuos y no encontraron actividad hemolítica (véase también Makkar y Becker, 1997 y Gidamis et al., 2003).

En el contexto de los factores antinutritivos, cabe regresar al tema del oxalato de calcio. El consumo de espinacas, acelgas, betabel y otras verduras con un contenido elevado de oxalatos podría, sobre todo en combinación con otros factores, como un bajo consumo de agua y el consumo de altos niveles de carne, contribuir a la formación de cálculos renales (Finkelstein y Goldfarb, 2006). Por lo tanto, es esencial contar con detalles sobre los niveles de oxalatos en la moringa, sobre todo de los oxalatos solubles, que son los que pueden contribuir a la formación de cálculos. Aunque los oxalatos están en cantidades abundantes en todos los tejidos de la moringa (Olson, 2001a; Olson y Carlquist, 2001), Radek y Savage (2008) mostraron que las hojas de la planta contienen únicamente oxalatos no solubles. Estos oxalatos no solubles se excretan en las heces, por lo que la moringa no parece ser una planta que contribuya a la formación de cálculos renales, aún si se consume en altas cantidades. En resumen, además de contar con altos niveles de aminoácidos esenciales, vitaminas y antioxidantes, los estudios indican que el consumo de la moringa no presenta riesgo, pues contiene niveles bajísimos de factores antinutritivos.

Es necesario mencionar que algunos glucosinolatos pueden ser bociógenos al interferir con la función de la tiroides. En Moringa los glucosinolatos parecen ser elementos clave en la prevención del cáncer, por lo que resulta esencial saber si la planta contiene glucosinolatos bociógenos. Es ampliamente conocido que las plantas de la familia Brassicaceae, parientes no tan lejanas de Moringaceae, contienen el glucosinolato progoitrina que, al hidrolizarse, libera la sustancia bociógena oxazolidonetiona. Además, muchos de los glucosinolatos formados alrededor de la molécula indola presentan actividad boció- 
gena. Sin embargo, en todos los estudios del contenido fitoquímico de la moringa, se ha encontrado que no contiene progoitrina y que los niveles de glucosinolatos indola son bajísimos (Fig. 2; Faizi et al., 1994, Guevara et al., 1999). Por lo tanto, no existe actualmente información que apunte a un riesgo bociógeno de la moringa. Sin embargo, existe la posibilidad de que algunos de los tiocianatos que contiene podrían presentar actividad bociógena en cantidades muy altas y mucho más allá de lo que se encontraría en una dieta normal, otra área potencial de investigación.

El papel de la moringa en la dieta y el desarrollo comunitario. La pregunta principal de la mayoría de los productores y consumidores de la moringa es si vale la pena cultivarla y consumirla. Con base en esta breve revisión de la literatura y de nuestros estudios, todo parece indicar que el consumo de la moringa ofrece numerosos beneficios nutritivos y contribuye a la prevención de enfermedades. Es importante señalar que sin estudios clínicos en seres humanos es imposible especificar las dosis necesarias para producir algún efecto benéfico, como, por ejemplo, la regulación de los niveles de glucosa en la sangre; sin embargo, parece probable que el consumo en dosis muy pequeñas aportaría poco o nada y para percibir beneficios sería necesario consumir la planta como verdura. Para establecer dosis precisas, el estudio clínico de los beneficios de la moringa es un campo abierto que aportará información muy útil. Sin embargo, la ausencia de esta información no debe ser razón para no consumir la planta, ya que consumimos verduras y frutas todos los días sin la necesidad de guiar nuestra elección con información derivada de estudios clínicos. Además, los beneficios potenciales son muchos y por sus niveles bajísimos de sustancias antinutricionales no se encuentran en este momento argumentos en contra del consumo de la planta. Por lo tanto, Moringa oleifera es una opción muy atractiva para ser cultivada en las comunidades tropicales secas de México y de otras partes de Latinoamérica, así como un alimento nutritivo y benéfico que se podría comercializar en una gama amplia de presentaciones. Si bien el conocimiento acerca de la utilidad de la moringa existe, todavía falta mucha investigación. Queda claro que para alcanzar su mejor aprovechamiento será necesario conocer la diversidad genética de la planta y cómo varían los parámetros de interés, tales como contenido de proteína o actividad antioxidante, entre linajes genéticos y entre ambientes.

Si bien la moringa claramente ofrece muchos beneficios potenciales, es necesario reconocer tanto los límites de lo que ofrece la planta como los límites de nuestro conocimiento, de tal suerte que el uso de la planta sea lo más congruente posible con lo que nos ofrece. Por ejemplo, el consumo de moringa como suplemento alimenticio parecería no tener sentido desde el punto de vista de su contenido de pro- teína, vitaminas y minerales, porque las cantidades que se consumen en "suplementos," tales como píldoras, pastillas, extractos, etc., no alcanzan niveles relevantes para el cuerpo. El consumo de la moringa como verdura parecería ser necesario para percibir sus beneficios alimenticios plenamente, ya sea en forma seca o aún mejor fresca. Aquí se ofrece un resumen breve de la gran cantidad de afirmaciones que se hacen acerca de las propiedades medicinales de la moringa, sin avalar el consumo de suplementos como cápsulas o concentrados. El criterio que se comparte con el de la mayoría de la comunidad científica es que el desarrollo de alimentos o suplementos nutricionales (como los nutracéuticos) tiene que seguir un camino diseñado explícitamente para producir conocimiento sobre la fitoquímica, el metabolismo y la farmacología (véase también Talalay and Talalay, 2001; Fahey and Kensler, 2007). De ser de otra forma, nunca será posible identificar los riesgos y especificar las dosis necesarias para lograr un beneficio dado.

\section{Conclusión}

Por sus características, la moringa puede utilizarse para que las comunidades en México y otras partes de América Latina no sólo mejoren su alimentación, sino que gestionen su sostenibilidad. El encarecimiento de los combustibles de origen fósil, debido a la explotación de yacimientos no convencionales y la menor productividad de varias regiones (Campbell, 2005), provocará, además de los problemas económicos y financieros que ya experimentamos, que el transporte de agua, alimentos y fármacos a los asentamientos humanos sea cada vez más costoso. Ante esta situación, se plantea como alternativa satisfacer el mayor número posible de las necesidades básicas con los recursos disponibles localmente (Odum y Odum, 2006). El mayor precio a pagar para traer alimentos y otros recursos desde sitios lejanos de producción implicará que si una comunidad acaba con un recurso local, ya no será posible reemplazarlo (Jepson, 2004). Por lo tanto, una respuesta ante las complicaciones que traerá el encarecimiento e inevitable agotamiento de los hidrocarburos es el manejo prudente de los recursos vegetales maderables y no maderables de tal forma que puedan ser utilizados para diversos fines (Páez, 2010). La capacidad de la moringa de ofrecer alimentos nutritivos para personas y animales, así como aceite comestible de alta calidad y una gama de otras aplicaciones, la vuelve un elemento sumamente importante para el establecimiento de comunidades sostenibles en el trópico seco, tanto en las áreas rurales como en las ciudades. Esto requiere el desarrollo no sólo de plantaciones en el campo, sino proyectos de agricultura urbana y periurbana en combinación con el aprovechamiento de otras especies. 


\section{Agradecimientos}

Agradecemos a Armando Páez, Julieta Rosell, Leonardo Alvarado, Vanessa Rojas, Gabriela Montes, Laura Trejo, Isaura Fernández y Alfredo Saynes por sus valiosos comentarios sobre versiones anteriores y a Magda Carvajal por su ayuda con la traducción de los nombres de las sustancias químicas. JWF agradece el apoyo del NIH (USA): R01 CA93780 y la Lewis B. and Dorothy Cullman Foundation.

\section{Literatura citada}

Allwood, M. C. y J. H. Plane. 1984. The degradation of vitamin A exposed to ultraviolet radiation. International Journal of Pharmaceutics 19:207-213.

Amaglo, N. K., R. N. Bennett, R. B. Lo Curto, E. A. S. Rosa, V. Lo Turco, A. Giuffrida, A. Lo Curto, F. Crea y G. M. Timpo. 2010. Profiling selected phytochemicals and nutrients in different tissues of the multipurpose tree Moringa oleifera L. [sic], grown in Ghana. Food Chemistry 122:1047-1054.

Anderson, D. M. W., P. C. Bella, M. C. L. Gill, F. J. McDougall y C. G. A. McNab. 1986. The gum exudates from Chloroxylon swietenia, Sclerocarya caffra, Azadirachta indica and Moringa oleifera. Phytochemistry 25:247-249.

Anwar, F., A. Siddiq, S. Iqbal y M. Rafique. 2007. Stabilization of sunflower oil with Moringa oleifera leaves under ambient storage. Journal of Food Lipids 14:35-49.

APG (Angiosperm Phylogeny Group). 2009. An update of the Angiosperm Phylogeny Group classification for the orders and families of flowering plants: APG III. Botanical Journal of the Linnean Society 161:105-121.

Bennett, R. N., F. A. Mellon, N. Foidl, J. H. Pratt, M. S. DuPont, L. Perkins y P. A. Kroon. 2003. Profiling glucosinolates and phenolics in vegetative and reproductive tissues of the multipurpose trees Moringa oleifera L. (Horseradish tree) and Moringa stenopetala L. Journal of Agricultural and Food Chemistry 51:3546-3553.

Bharali, R., J. Tabassum y M. R. H. Azad. 2003. Chemomodulatory effect of Moringa oleifera, Lam, on hepatic carcinogen metabolizing enzymes, antioxidant parameters and skin papillomagenesis in mice. Asian Pacific Journal of Cancer Prevention 4:131-139.

Campbell, C. J. 2005. Oil crisis. Multi-Science, Essex. 397 p.

Das, B. R., P. A. Kurup y P. L. Narasimha-Rao. 1954. Antibiotic principle from Moringa pterygosperma. Naturwissenschaften 41:66.

Das, B. R., P. A. Kurup y P. L. Narasimha-Rao. 1957a. Antibiotic principle from Moringa pterygosperma. Part VII. Antibacterial activity and chemical structure of compounds related to pterigospermin. Indian Journal of Medical Research 45:191196.
Das, B. R, P. A. Kurup, P. L. Narasimha-Rao y A. S. Ramaswamy. 1957b. Antibiotic principle from Moringa pterygosperma. Part VIII. Some pharmacological properties and in vivo action of pterigospermin and related compounds. Indian Journal of Medical Research 45:197-206.

de la Barrera, E. 2008. Recent invasion of buffel grass (Cenchrus ciliaris) of a natural protected area from the southern Sonoran Desert. Revista Mexicana de Biodiversidad 79:385-392.

Doerr, B., K. L. Wade, K. K. Stephenson, S. B. Reed y J. W. Fahey. 2009. Cultivar effect on Moringa oleifera glucosinolate content and taste: a pilot study. Ecology of Food and Nutrition 48:199-211.

Eilert, U., B. Wolters y A. Nahrstedt. 1981. The antibiotic principle of seeds of Moringa oleifera and Moringa stenopetala. Planta Medica 42:55-61.

Fahey, J. W. 2005. Moringa oleifera: a review of the medical evidence for its nutritional, therapeutic, and prophylactic properties. Part 1. Trees for Life Journal 1:5. Disponible en http://www.TFLJournal.org/article.php/20051201124931586; última consulta: 14.X.2010.

Fahey, J. W., X. Haristoy, P. M. Dolan, T. W. Kensler, I. Scholtus, K. K. Stephenson, P. Talalay y A. Lozniewski. 2002. Sulforaphane inhibits extracellular, intracellular, and antibiotic-resistant strains of Helicobacter pylori and prevents benzo[a]pyrene-induced stomach tumors. Proceedings of the National Academy of Sciences USA 99:7610-7615.

Fahey, J. W. y T. W. Kensler. 2007. Role of dietary supplements/ nutraceuticals in chemoprevention through induction of cytoprotective enzymes. Chemical Research in Toxicology 20:527-576.

Fahey, J. W., A. T. Dinkova-Kostova y P. Talalay. 2004. The "Prochaska" microtiter plate bioassay for inducers of NQO1. In Methods in enzymology, vol. 382, Parte B, H. Sies y L. Packer (eds.). Elsevier Science, San Diego, California. p. 243258.

Fahey, J. W., A. T. Zalcmann y P. Talalay. 2001. The chemical diversity and distribution of glucosinolates and isothiocyanates among plants. Phytochemistry 56:5-51. [corrigendum: Phytochemistry 59:237].

Faizi, S., B. S. Siddiqui, R. Saleem, S. Siddiqui, K. Aftab y A. H. Gilani. 1994. Isolation and structure elucidation of new nitrile and mustard oil glycosides from Moringa oleifera and their effect on blood pressure. Journal of Natural Products 57:12561261.

Fernández, I. V. 2010. Moringa oleifera y su impacto en el estado nutricional de vitamina A, hierro y zinc en preescolares: Estudio piloto. Tesis maestría, Centro de Investigación en Alimentación y Desarrollo, Hermosillo, Sonora.

Ferreira, P. M. P., D. F. Farias, J. T de A. Oliveira y A. de F. U. Carvalho. 2008. Moringa oleifera: bioactive compounds and nutritional potential. Revista de Nutrição Campinas 21:431437. 
Finkelstein, V. A. y D. S. Goldfarb. 2006. Strategies for preventing calcium oxalate stones. Canadian Medical Association Journal 174:1407-1409.

Freiberger, C. E., D. J. Vanderjagt, A. Pastuszyn, R. S. Glew, G. Mounkaila, M. Millson y R. H. Glew. 1998. Nutrient content of the edible leaves of seven wild plants from Niger. Plant Foods for Human Nutrition 53:57-69.

Fuglie, L. J., ed. 2001. The miracle tree: The multiple attributes of moringa. Technical Centre for Agricultural and Rural Cooperation, Wageningen/ Church World Service, New York. $172 \mathrm{p}$.

Galan, M. V., A. A. Kishan y A. L. Silverman. 2004. Oral broccoli sprouts for the treatment of Helicobacter pylori infection: a preliminary report. Digestive Disease Science 49:1088-1090.

Gidamis, A. B., J. T. Panga, S. V. Sarwatt, B. E. Chove y N. B. Shayo. 2003. Nutrient and antinutrient contents in raw and cooked young leaves and immature pods of Moringa oleifera, Lam. Ecology of Food and Nutrition 42:399-411.

Guevara, A. P., C. Vargas, H. Sakurai, Y. Fujiwara, K. Hashimoto, T. Maoka, M. Kozuka, Y. Ito, H. Tokuda y H. Nishino. 1999. An antitumor promoter from Moringa oleifera Lam. Mutation Research 440:181-188.

Haines, H. H. 1922. Botany of Bihar and Orissa. Part III. Calyciflorae. Adlard \& Son \& West Newman, London. 316 p.

Haristoy, X., J. W. Fahey, I. Scholtus y A. Lozniewski. 2005. Evaluation of antimicrobial effect of several isothiocyanates on Helicobacter pylori. Planta Medica 71:326-330.

Hartwell, J. L. 1967-1971. Plants used against cancer: a survey. Lloydia 30-34.

Janzen, D. 1988. Tropical dry forests. The most endangered major tropical ecosystem. In Biodiversity, E.O. Wilson (ed.). National Academy of Sciences/ Smithsonian Institution, Washington D. C. p. 130-137.

Jepson, E. 2004. The adoption of sustainable development policies and techniques in U.S. cities: how wide, how deep, and what role for planners? Journal of Planning Education and Research 23:229-241

Kar, A., B. K. Choudhary y N. G. Bandyopahyay. 2003. Comparative evaluation of hypoglycaemic activity of some Indian medicinal plants in alloxan diabetic rats. Journal of Ethnopharmacology 84:105-108.

Keraudren, M. y J. B. Gillett. 1963. Sur le type du Moringa oleifera Lam., et la valeur de ce binôme. Bulletin de la Société Botanique de France 110:316-318.

Kjaer, A., O. Malver, B. El-Menshawi y J. Reisch. 1979. Isothiocyanates in myrosinase-treated seed extracts of Moringa peregrina. Phytochemistry 18:1485-1487.

Kumar, N. A. y L. Pari. 2003. Antioxidant action of Moringa oleifera Lam. (drumstick) against antitubercular drugs induced lipid peroxidation in rats. Journal of Medicinal Food 6:255259.

Kurup, P. A. y P. L. Narasimha-Rao. 1952. Antibiotic principle from Moringa pterygosperma. Part I. Journal of the Indian Institute of Science 34:219-227.

Kurup, P. A. y P. L. Narasimha-Rao. 1954a. Antibiotic principle from Moringa pterygosperma. Part II. Chemical nature of pterigospermin. Indian Journal of Medical Research 42:85-95.

Kurup, P. A. y P. L. Narasimha-Rao. 1954b. Antibiotic principle from Moringa pterygosperma. Part IV. The effect of addition of vitamins and amino acids on the anti-bacterial activity of pterigospermin. Indian Journal of Medical Research 42:101107.

Kurup, P. A. y P. L. Narasimha-Rao. 1954c. Antibiotic principle from Moringa pterygosperma. Part V. Effect of pterigospermin on the assimilation of glutamic acid by Micrococcus pyogenes var. aureus. Indian Journal of Medical Research 42:109-114.

Luqman, S., S. Kaushik, S. Srivastava, R. Kumar, D. U. Bawankule, A. Pal, M. P. Darokar y S. P. S. Khanuja. 2009. Protective effect of medicinal plant extracts on biomarkers of oxidative stress in erythrocytes. Pharmaceutical Biology 47:483-490.

Makkar, H. P. S. y K. Becker. 1996. Nutritional value and antinutritional components of whole and ethanol extracted Moringa oleifera leaves. Animal Feed Science and Technology 63:211-228.

Makkar, H. P. S. y K. Becker. 1997. Nutrients and antiquality factors in different morphological parts of the Moringa oleifera tree. Journal of Agricultural Science 128:311-322.

Martínez, M. 1959. Plantas útiles de la flora mexicana. Botas, México, D. F. 621 p.

Mehta, L. K., R. Balaraman, A. H. Amin, P. A. Bafna y O. D. Gulati. 2003. Effects of fruits of Moringa oleifera on the lipid profile of normal and hypercholesterolaemic rabbits. Journal of Ethnopharmacology 86:191-195.

Miles, L., A. C. Newton, R. S. DeFries, C. Ravilious, I. May, S. Blyth, V. Kapos y J. E. Gordon. 2006. A global overview of the conservation status of tropical dry forests. Journal of Biogeography 33:491-505.

Morton, J. F. 1991. The horseradish tree, Moringa pterygosperma (Moringaceae) - A boon to arid lands? Economic Botany 45:318-333.

Murakami, A., Y. Kitazono, S. Jiwajinda, K. Koshimizu y H. Ohigashi. 1998. Niaziminin, a thiocarbamate from the leaves of Moringa oleifera, holds a strict structural requirement for inhibition of tumor-promoter-induced Epstein-Barr virus activation. Planta Medica 64:319-323.

Nambiar, V. S. y S. Seshadri. 2001. Bioavailability trials of b-carotene from fresh and dehydrated drumstick leaves (Moringa oleifera) in a rat model. Plant Foods for Human Nutrition 56:83-95.

Ndong, M., M. Uehara, S. Katsumata y K. Suzuki. 2007. Effects of oral administration of Moringa oleifera Lam on glucose tolerance in Goto-Kakizaki and Wistar rats. Journal of Clinical Biochemistry and Nutrition 40:229-233. 
Odum, H. T. y E. C. Odum. 2006. The prosperous way down. Energy 31:21-32.

Olson, M. E. 2001a. Stem and root anatomy of Moringa (Moringaceae). Haseltonia 8:56-96.

Olson, M. E. 2001b. Introduction to the Moringa Family. In The miracle tree: The multiple attributes of Moringa, L. J. Fuglie (ed.). Technical Centre for Agricultural and Rural Cooperation, Wageningen/ Church World Service, New York. p. 11-28.

Olson, M. E. 2002a. Combining data from DNA sequences and morphology for a phylogeny of Moringaceae. Systematic Botany 27:55-73.

Olson, M. E. 2002b. Intergeneric relationships within the Caricaceae-Moringacecae clade (Brassicales), and potential morphological synapomorphies of the clade and its families. International Journal of Plant Sciences 163:51-65.

Olson, M. E. 2003. Ontogenetic origins of floral bilateral symmetry in Moringaceae. American Journal of Botany 90:49-71.

Olson, M. E. 2010. Moringaceae. In Flora of North America North of Mexico, vol. 7, Flora of North America Editorial Committee (eds.). Flora of North America Association, New York y Oxford. p. 167-169.

Olson, M. E. y S. G. Razafimandimbison. 2000. Moringa hildebrandtii: A tree extinct in the wild but preserved by indigenous horticultural practices in Madagascar. Adansonia sér. 3 22:217-221.

Olson, M. E. y S. Carlquist. 2001. Stem and root anatomical correlations with life form diversity, ecology, and systematics in Moringa (Moringaceae). Botanical Journal of the Linnean Society 135:315-348.

Ortega-Nieblas, M., L. Vázquez-Moreno y M. R. RoblesBurgueño. 1996. Protein quality and antinutritional factors of wild legume seeds from the Sonoran Desert. Journal of Agricultural and Food Chemistry 44:3130-3132.

Páez, A. 2010. Energy-urban transition: the Mexican case. Energy Policy 38:7226-7234.

Palada, M. C. 1996. Moringa (Moringa oleifera Lam.): A versatile tree crop with horticultural potential in the subtropical United States. HortScience 31:794-797.

Piperno, D. R., A. J. Ranere, I. Holst, J. Iriarte y R. Dickau. 2009. Starch grain and phytolith evidence for early ninth millennium B.P. maize from the Central Balsas River Valley, Mexico. Proceedings of the National Academy of Sciences of the USA. 106:5019-5024.

Radek, M. y G. P. Savage. 2008. Oxalates in some Indian green leafy vegetables. International Journal of Food Sciences and Nutrition 59:246-260.

Rao, K. N. V., V. Gopalakrishnan, V. Loganathan y S. Shanmuganathan. 1999. Antiinflammatory activity of Moringa oleifera Lam. Ancient Science of Life 18:195-198.

Reddy, V., A. Urooj y A. Kumar. 2003. Evaluation of antioxidant activity of some plant extracts and their application in biscuits.
Food Chemistry 90:317-321.

Reyes, N., E. Spörndly e I. Ledin. 2006. Effect of feeding different levels of foliage of Moringa oleifera to creole dairy cows on intake, digestibility, milk production and composition. Livestock Science 101:24-31.

Richter, N., P. Siddhuraju y K. Becker. 2003. Evaluation of nutritional quality of moringa (Moringa oleifera Lam.) leaves as an alternative protein source for Nile tilapia (Oreochromis niloticus L.). Aquaculture 217:599-611.

Sampson, W. 2005. Studying herbal remedies. New England Journal of Medicine 353:337-339.

Seshadri, S., M. Jain y D. Dhabhai. 1997. Retention and storage stability of beta-carotene in dehydrated drumstick leaves (Moringa oleifera). International Journal of Food Sciences and Nutrition. 48:373-379.

Shaw, B. P. y P. Jana. 1982. Clinical assessment of Sigru (Moringa oleifera Lam) on Mutrakrichra (lower urinary tract infection). Nagarjun 25:231-235.

Siddhuraju, P. y K. Becker. 2003. Antioxidant properties of various solvent extracts of total phenolic constituents from three different agroclimatic origins of Drumstick Tree (Moringa oleifera Lam.) leaves. Journal of Agricultural and Food Chemistry 51:2144-2155.

Singh, B. N., B. R. Singh, R. L. Singh, D. Prakash, R. Dhakarey, G. Upadhyay y H. B. Singh. 2009. Oxidative DNA damage protective activity, antioxidant and anti-quorum sensing potentials of Moringa oleifera. Food and Chemical Toxicology 47:1109-1116.

Stephenson, K. K. y J. W. Fahey. 2004. Development of tissue culture methods for the rescue and propagation of endangered Moringa spp. germplasm. Economic Botany 58:s116-s124.

Talalay, P. y P. Talalay. 2001. The importance of using scientific principles in the development of medicinal agents from plants. Academic Medicine 76:238-247.

Thurber, M. y J. W. Fahey. 2009. Adoption of Moringa oleifera to combat under-nutrition viewed through the lens of the 'Diffusion of Innovations' theory. Ecology of Food and Nutrition 48:212-225.

USAID (U. S. Agency for International Development). 2006. Fact sheet: nonfat dry milk. Disponible en http://www.usaid. gov/our_work/humanitarian_assistance/ffp/crg/downloads/ fsnfdrymilk.pdf; última consulta: 7.IX.2010.

Verdcourt, B. 1985. A synopsis of Moringaceae. Kew Bulletin 40:1-23.

Villaseñor R., J. L. y F. J. Espinosa G. 1998. Catálogo de malezas de México. Universidad Nacional Autónoma de México, México, D. F. 448 p.

Yanaka, A., S. Zhang, M. Yamamoto y J. W. Fahey. 2005. Daily intake of sulforaphane-rich broccoli sprouts improves gastritis in H. pylori-infected human subjects. Cancer Epidemiology Biomarkers and Prevention 14: 14:2754s. 